\title{
Acute Tubular Necrosis
}

National Cancer Institute

\section{Source}

National Cancer Institute. Acute Tubular Necrosis. NCI Thesaurus. Code C34749.

Acute renal failure caused by the cell death of the renal tubules. Causes include nephrotoxins, cytotoxic drugs, and antibiotics. 